\title{
On the relativity of quantitative model results for policy purposes in the social sciences.*
}

\author{
Paul G.C. Mensink. $\ddagger$ \\ Environmental Economics Group \\ Department of Social Sciences \\ Wageningen University.
}

July, 2000

\begin{abstract}
This paper discusses the potential sources of misinterpretation of quantitative model results by policy makers. It is argued that model results are relative, in other words, the correct interpretation of the results depends on (1a) the policy maker for whom the model was meant for as a decision support system - as well as - (1b) whether the model is meant to be descriptive or prescriptive, and (2), in case of stochastic parameters in the model, whether it is the actors or the policy maker that experience uncertainty.

It is concluded that the chance of misinterpretation can be reduced if the model developer explicitly describes key characteristics of the policy maker the model results are meant for. Examples of potential misinterpretation of model results are given for the field of greenhouse gas emission control.
\end{abstract}

JEL classification: D78, D83, Q28.

Keywords: Climate change; Decision criterium; Environmental policy; Modeling; Uncertainty.

*Please refer to as Mensink, P.G.C. (2000) "On the relativity of quantitative model results in the social sciences" Wageningen Economic Paper 12, Department of Social Sciences, Wageningen University, Wageningen, The Netherlands. Internet: http://www.wau.nl/wub/wep/wephome.htm.

${ }^{\diamond}$ Environmental Economics Group, Department of Social Sciences, Wageningen University, PO Box 8130, 6700 EW Wageningen, The Netherlands. E-mail address: paul.mensink@alg.shhk.wau.nl. Telephone: +31317 427484. Fax: +31317484933.

${ }^{\ddagger}$ Discussions with and comments by Rob Dellink, Leen Hordijk, Ekko van Ierland, Carolien Kroeze, Walter Ruijgrok, and Stephan Slingerland improved the quality of this paper significantly. 


\section{Introduction}

Research on the issue of global warming is usually meant to support decision-making, either directly or indirectly. Governments make decisions on whether, where, when and how to abate greenhouse gas emissions. They have used the results from social and natural sciences to base their decisions on (Bader, 1998, p.41) and will most likely use them in the future. However, "the relationship between analysis and policy is often characterized by problems of misunderstanding and mistrust between analysts and decision makers' (Robinson, 1992, p.147). This paper discusses potential sources of misinterpretation of quantitative model results by policy makers. It focuses on the relativity of model results with respect to the particular policy maker who will use the results.

The pitfalls associated with interpretation of model results for policy making have been discussed by Clark and Majone (1985), who design a classification of critical criteria used in actual cases of scientific inquiry in policy contexts.

In addition there are a number of studies that address the caveats associated with modeling for global warming related policy making. O'Riordan (1997) has pointed at the potential misinterpretation with regard to descriptive and prescriptive approaches in the IPCC second assessment (IPCC, 1996). Parson (1996, p.320) discusses fundamental difficulties with respect to the interpretation of projections of long-term human choices. Manne (1995) pointed at the importance to make a distinction between prescriptive and descriptive reasoning by means of a numerical experiment. Also, Azar (1998) identifies four significant, potentially value-laden choices made by economic modelers in the context of global warming. Three out of these four choices (how to evaluate costs, the choice of the discount rate, and the choice of the decision criterion) give rise to problems with the interpretation of model results. These problems are associated with the relativity of model results with respect to the policy maker who will use the results.

This paper formalizes and generalizes these caveats into two categories of potential misinterpretation by decision-makers of the results of quantitative modeling in research fields where human behavior, either individually or collectively, plays a role. The first category is assumptions with regard to the preferences and decision-making power of the policy maker. Related to this issue is the descriptive or prescriptive nature of model. The second category is assumptions with regard to the subjectivity of uncertainty. These two categories are of particular relevance for the global warming issue. The first category is relevant because the global warming issue involves many stakeholders (individuals, regions, sectors and generations) who potentially have different objectives, and levels of decision-making power ${ }^{1}$ and therefore will have to interpret results from prescriptive modeling efforts differently. The second category is relevant because decision-making is hampered by the uncertainty of scientists and decision-makers with regard to the necessity and consequences of decisions

\footnotetext{
${ }^{1}$ Parson (1995, p.463), declared - expressed in the terms used in this paper - the problem of nonrepresentative decision-making power is one of "three significant representational weaknesses [in environmental policy making] of decadal-scale emission trends."
} 
on greenhouse gas emission reductions, partly originating from the long time scale of the issue and the chaotic nature of the climate system.

More specifically, this paper discusses the following issues: (1a) What is the influence on the interpretation of model results of the preference and the decision-making power of the decision-maker for whom the model was developed? And related, (1b) what is the relevance of the distinction between prescriptive and descriptive models for the interpretation of model results? (2) What is the relevance of the distinction between uncertainty experienced by a model user on the one hand, and the actors - whose behaviors are described in the model - on the other hand, for interpretation of model results?

To prevent the discussion from becoming too complicated, the discussion is based on the assumption that there is only one model. This assumption conflicts with the reality of the global warming issue where many different policy makers are confronted with various quantitative models to support their decision-making process (Jaeger et al., 1998). However, understanding well the case with only one model is a necessary pre-condition for understanding the situation with multiple models.

After introducing some key notions, definitions of descriptive and prescriptive uses of a quantitative model for policy purposes are given to highlight the differences between the two approaches (section 2). Next, examples of potential misinterpretation relating to the descriptive and prescriptive use of models are given (section 3). Then, an example of potential misinterpretation of the attribution of uncertainty is given (section 4). Finally, conclusions from this analysis are drawn, their relevancy for climate research is highlighted, and corollaries for the presentation of research results are discussed (section 5).

\section{Key notions and definitions}

\section{$2.1 \quad$ Key notions}

In order to define the descriptive and prescriptive use of quantitative model results for policy purposes the following key notions are introduced. Although the description of the notions are limited to this paper, they are formulated in a manner that keeps 'as close as possible' to the different ways these concepts are generally used in policy modeling.

Model A set of relations that associates values with endogenous variables given exogenous variables. This set of relations forms a function and will be symbolized by either $f_{M}(\cdot)$ or $f_{N}(\cdot)$, to be abbreviated in the text to $M$ respectively $N$. A more elaborate definition of model is given in section 2.2 .

Policy maker Person, group of persons, or an organization that uses a quantitative model as a basis for decision-making. They are symbolized with $P$ or $Q$. 
Actor Model component that describes the behavior of an agent, government, or individual who selects his decisions endogenously in the model, thereby representing the agent, government, or individual. These decisions are selected given an implicitly or explicitly formulated preference criterium in concert with physical and/or economic constraints.

Preference function (of an actor or policy maker) The preference function $g_{P}$ of a policy maker or actor $P$ (e.g. a utility function) associates a value to a decision and its consequences. The higher values of $g_{P}$, the more preferable the outcome.

Decision space (of an actor or policy maker) The set of options a policy maker can choose from. Here symbolized with $D_{P}$ or $D_{Q}$.

In following sections, the term 'decision space' of a policy maker will represent its 'decisionmaking power'. These terms are not equivalent. The freedom to choose from different options, however, is strongly related to the extent to which the policy maker has influence on his environment. The use of the term 'decision-making power' is avoided in the following because of the broad spectrum of potential interpretations.

\subsection{Using a model as descriptive or prescriptive}

Assume now that policy maker $P$ uses model $M$. The relations in $M$ are described by the function $f_{M}$. Thus the relations between the inputs of model $M$ and the output of model $M$ is described by the function $f_{M}$.

This discussion begins by presenting the descriptive use of a model $M$. The function $f_{M}$ which describes the relations in model $M$ - associates output vector $r \in O$ relevant for policy maker $P$, to $P$ 's decisions $u \in D_{P}$ and variables $\bar{u} \in \bar{D}_{P} \cdot{ }^{2}$ Variables $\bar{u} \in \bar{D}_{P}$ represent exogenous variables that can not be influenced by the policy maker. In mathematical terms ${ }^{3}$ :

$$
f_{M}: D_{P} \times \bar{D}_{P} \rightarrow O
$$

where $O$ is the set of output vectors. With the expression "Policy maker $P$ uses model $M$ as descriptive" the following is meant: policy maker $P$ evaluates the potential decisions in his decision space and picks the one most preferable by means of model $M$. Or in mathematical terms, the policy maker will try to find the most preferable decision $u_{M}^{*} \in D_{P}{ }^{4}$ for which the following relation holds:

\footnotetext{
${ }^{2}$ For an overview of the variables used here and their interpretation see Table 1.

${ }^{3} f: A \times B \rightarrow C$ means that the function $f$ maps an element of set $A$ and an element of set $B$ on an element in set $C$.

${ }^{4}$ For reasons of convenience it is assumed that there is one and only one solution for all optimization problems stated in this paper.
} 


$$
g_{P}\left(u_{M}^{*}, \bar{u}, f_{M}\left(u_{M}^{*}, \bar{u}\right)\right) \geq g_{P}\left(u, \bar{u}, f_{M}(u, \bar{u})\right) \quad \text { for all } u \in D_{P}
$$

given a $\bar{u}$, and given $g_{P}: D_{P} \times \bar{D}_{P} \times O \rightarrow \mathcal{R}$, the preference function of policy maker $\mathrm{P}^{5}$. With the expression "Policy maker $Q$ uses the model $N$ as prescriptive" it is meant that policy maker $Q$ uses the model $N$ to select a most preferable decision in the following way: the model $N$ selects $u_{N}^{*}=f_{N}(\bar{u})$ as a most preferable decision, where $f_{N}(\bar{u})$ describes the relations in model $\mathrm{N}$

$$
f_{N}: \bar{D}_{Q} \rightarrow D_{Q}
$$

and

$$
\begin{aligned}
f_{N}(\bar{u})= & \arg \max _{u} g_{Q}(u, \bar{u}, x) \\
& \text { s.t. } h_{N}(u, \bar{u}, x) \leq 0 \\
& u \in D_{Q}
\end{aligned}
$$

where $x \in X$ is a vector of endogenous variables necessary to describe the relations in model $N$, and $g_{Q}: D_{Q} \times \bar{D}_{Q} \times X \rightarrow \mathcal{R}$ is a function that represents the preferences of policy maker $Q$, and $h_{N}(\cdot)$ represents constraints on combinations of $u, \bar{u}$ and $x$, originating from physical, technical or any other laws, rules or relationships. ${ }^{6}$

\section{Potential misinterpretations of quantitative model results for policy purposes}

In the following section, the relevance of (1a) the preferences of the policy maker and the decision-making power of the policy maker, and $(1 \mathrm{~b})$ the difference between descriptive and prescriptive model use for the interpretation of the model results are discussed by means of examples of potential misinterpretations of model results.

\subsection{Policy maker: preferences and decision space}

Preferences. Assume a model is meant to be used as prescriptive by policy maker $P_{1}$ as described in section 2.2. When the decision space and the preferences of $P_{1}$ and another policymaker $P_{2}$ are equivalent, $P_{2}$ may interpret the results as descriptive. When these preferences are not equivalent the results may not necessarily be interpreted as prescriptive and could lead to unintended decisions.

\footnotetext{
${ }^{5} \mathcal{R}$ is the set of real numbers

${ }^{6}$ The function $\arg \max _{u} g(u)$ has the value of $u$ for which $g(u)$ attains the highest value.
} 
Applied example: A prescriptive model that is developed for a policy maker representing the OECD countries to derive for him efficient emission reductions strategies might result in inefficient or infeasible emission reduction strategies if it is used by a policy maker representing the Danish government. In this case the preference of the policy maker representing the OECD countries in total might be different from the policymaker representing Denmark. This difference in preferences might, for example, be caused by the phenomenon that at the higher income levels of Denmark households, a representative policy maker will tend to choose more environmental quality than an OECD policy maker (e.g., McConnell, 1997, p.383) .

The interpretation of prescrictive model results is likely to depend on the preferences of the policy maker the model was developed for. In short, the model results are relative with respect to the preferences of the policy maker. Also, when a model is meant to be used as prescriptive it would be wise either (i) to state explicitly for which policy maker its results are intended as prescriptive, or (ii) to formulate explicitly the preferences of policy makers for whom the model results are to be interpreted as prescriptive, together with a warning for other policy makers that application of the model results might not be in their interest. $^{7}$

Decision space The following example relates to what is described as 'one of the hardest, most important, and most enduring problems of doing assessment well'(Parson, 1996, pp.320,324)

Assume the decision space $D_{P}$ of policy maker $P$ in model $M$ is smaller then his decision space $D_{P}^{\prime}$ in reality (e.g. $D_{P} \subset D_{P}^{\prime}$ ). Let model $M$ be a prescriptive model with respect to policy maker $P$ that has been developed to generate optimal $\mathrm{CO}_{2}$ emission reduction strategies, where the costs of new renewable energy technologies are exogenous. But, suppose that policy maker $P$ has the decision-making power to reduce these costs significantly through research and the installation of subsidies (e.g., Neij, 1997, p.1105). Then, model $M$ might not prescribe the most preferable decisions. In fact, the decisions generated with the model would likely be biased towards the extrapolation of historic trends, 'lessening the probability of developing creative policy in response to present problems' (Robinson, 1992, p.148).

The same kind of argument holds for the descriptive use of a model, for example the MERGE2 model (Manne, 1995), when the decision space of the policy maker for whom the model was developed is smaller in the model than it is in reality.

This example points at that model results can be relative with respect to the decision space of the policy maker for whom the model was developed.

From this example we also learn that significant misinterpretations of results may be prevented when model developers explicitly state both for which policy maker the model is

\footnotetext{
${ }^{7}$ In some versions of the RAINS model (Alcamo, Shaw and Hordijk, 1990) the model user is given the opportunity to select an objective function from a set of pre-formulated objective functions (Hordijk, 1997). This opportunity reduces the chance of a mismatch with the preferences function of the model user.
} 
developed, and what the appropriate decision-making power of the policy makers that use the model has been assumed.

\subsection{Descriptive versus prescriptive.}

Assume model $M$ is developed as a descriptive model with the purpose to support decisionmaking for policy maker $P$. Assume results from $M$ are interpreted as prescriptive by $P$. This interpretation might result in non-intended solutions for policy maker $P$ : decisions that describe observed behavior properly, might not be optimal strategies. ${ }^{8}$

Applied example: Assume model $M$ is used as a descriptive model that serves to support decision-making in the field of global warming. To be specific, it is developed as a descriptive model for $P$, a hypothetical group of world leaders, and describes (i) timing of greenhouse gas emission reductions, and (ii) the level of greenhouse gas concentration stabilization that maximizes total discounted utility. Utility is a function of consumption. $M$ might, for example, be the MERGE2 model. In this example timing of greenhouse gas emission reduction strategies might describe observed behavior properly - or be consistent with observed behavior, but might not be optimal strategies for $P$. This situation could be caused by the public good-type ${ }^{9}$ character of taking care of future generations as argued by Pearce and Turner (1990, p.222), or it could be due to limits on the feasibility of existing intergenerational transfers, as argued by the researchers adopting the "prescriptive approach' that is described in in Arrow et al. (1996). ${ }^{10}$ Interpreting the results of $M$ as prescriptive might therefore result in non-optimal decisions, for example, in too much delay in the timing of reducing greenhouse gas emissions.

Approximately the same distinction between observed and intended behavior might hold for climate change damage estimates as those based on the concepts of 'willingness to pay' or 'willingness to accept' (e.g., Fankhauser et al., 1997): $P$, the hypothetical group of world leaders, could be willing to value damages in general and mortality risks in particular in regions outside their own higher than they show now in their actual choices with respect to money transfers to third world countries. Just like the willingness to support less wealthy countries was latent in Europe in the first half of the 20th century before the establishment and expansion of the European Union, interpreting the results of $M$ as prescriptive might result in non-optimal, for example too high levels of greenhouse gas emissions.

From these examples we can see that model results might have to be interpreted differently dependent on whether they are meant as descriptive or prescriptive. In short, model

\footnotetext{
${ }^{8}$ E.g. Stewart (1997) presents and analyses differences in results from descriptive and prescriptive studies of the value of weather forecasts.

${ }^{9} \mathrm{~A}$ public good is a good that is indivisible and non-excludable and therefore will be under supplied relative to the efficient level of supply. This efficient level of supply can be generated by a collective body, for example a government (Perman, Ma and McGilvray, 1996, p.101).

${ }^{10}$ For example there might be a collective decision to introduce a trust fund that gives future generations a share in environmental resources. This would relate to a lower discount rate when compared to a situation where the ownership is left entirely to the present generation (Gerlagh, 1998, ch. 4).
} 
results are relative with respect to the descriptive or prescriptive character of the model. We also recognize that misinterpretations of model results could be prevented if model developers state explicitly whether their results are meant to be descriptive or prescriptive for particular policy makers.

\subsection{Concluding remark}

Although the potential misinterpretation with respect to preferences, prescriptive vs. descriptive model use, and decision-making power are presented here separately for analytical purposes, they are strongly related: (i) prescriptive model use is by definition related to the preference of the policy maker, and (ii) decision-making power determines whether prefered goals are actually attainable.

\section{Policy makers, quantitative model results and un- certainty}

Now assume the parameter $\bar{u}$, which symbolizes factors that can not be influenced by the policy maker in section 2.2 , is not a deterministic exogenous variable, but a stochastic variable. Let $\overline{\mathbf{u}}$ be this stochastic variable.

Assume policy maker $Q$ uses model $M$ as a descriptive model and that the stochastic variable $\overline{\mathbf{u}}$ represents the factors that he can not influence.

There are two different ways of including this uncertainty, here represented by models $M$ and $N$ respectively.

Model M Policy maker $Q$ does not know the exact value $\overline{\mathbf{u}}$ will adopt, but he knows the distribution of $\overline{\mathbf{u}}$. Also the actors do not know the exact value $\overline{\mathbf{u}}$ will adopt, but they know the distribution of $\overline{\mathbf{u}}$. This model will be called model $M$.

Model $\mathbf{N}$ As in M, policy maker $Q$ does not know the exact value $\overline{\mathbf{u}}$ will adopt, but he knows the distribution of $\overline{\mathbf{u}}$. The actors, however, do know the exact value that $\overline{\mathbf{u}}$ will adopt. This model will be called model $N$.

It is significant to note that the two ways of including uncertainty as presented here are not exhaustive.

Model M. Without giving up generality with respect to the conclusions that can be drawn from this analysis, one can assume that the output of model $M$ is not stochastic. This would be the case, for example, when $M$ is described by the function $f_{M}$ where ${ }^{11}$

\footnotetext{
${ }^{11} E_{\overline{\mathbf{u}}}[\cdot]$ denotes the expected value function over distribution $\overline{\mathbf{u}} . \mathcal{P}[\overline{\mathbf{u}}]$ is the probability function of stochastic variable $\overline{\mathbf{u}}$. The expected value function over distribution $\overline{\mathbf{u}}$ is one of many functions that
} 


$$
\left.\begin{array}{rl}
f_{M}(u, \overline{\mathbf{u}})= & \{(v, x) \mid \\
& v_{i}=\arg \max _{z_{i}} E_{\overline{\mathbf{u}}}\left[g_{i}\left(z_{i}, x, u, \overline{\mathbf{u}}\right)\right] \\
& \text { s.t. } \mathcal{P}\left[h_{i}\left(z_{i}, x, u, \overline{\mathbf{u}}\right) \leq 0\right] \geq \alpha_{i}
\end{array}\right\} \quad i=1, \ldots, n
$$

In words, $f_{M}$ can be described as follows: in model $M$, actor $i$, with $i=1, \ldots, n$, maximizes the expected value of his preference function given stochastic constraints (equations 8), while interacting with other actors - for example in a market (equations 9) ${ }^{12}$. This is an example of a model with a stochastic input and non-stochastic output.

Examples of this type of model in the field of greenhouse gas emission scenarios - including its empirical application - can be seen in the work of (for one actor) Manne and Richels (1992, ch.4), and (for multiple actors) Manne and Olsen (1996), both under the name 'act then learn'. In both models damage potential associated with a given level of radiative forcing is stochastic.

The policy maker $Q$ now can pick the most preferable decision $u_{M}^{*}$ in the same way, mutatis mutandis, he did in section 2.2 by maximizing a preference function $g_{Q}(u, \overline{\mathbf{u}},(v, x))=$ $g_{Q}\left(u, \overline{\mathbf{u}}, f_{M}(u, \overline{\mathbf{u}})\right)$.

Model N. Assume $\overline{\mathbf{u}}$ has value $\bar{u}_{k}$ with respective probabilities $\pi_{k}, k=1, \ldots, l$. In model $N$, any actor observes the true value of $\overline{\mathbf{u}}$, being $\bar{u}_{k}$. The policy maker does not observe this value, and only knows the distribution of $\overline{\mathbf{u}}$. Now the output of $N, f_{N}(u, \overline{\mathbf{u}})$ is a stochastic variable itself. This is the case because from the point of view of the policy maker, actor $i$ makes the following decision

$$
\begin{array}{r}
v_{i k}=\quad \arg \max _{z_{i k}} g_{i}\left(z_{i k}, x_{k}, u, \bar{u}_{k}\right) \\
\text { s.t. } h_{i}\left(z_{i k}, x, u, \bar{u}_{k}\right) \leq 0
\end{array}
$$

with probability $\pi_{k}$. Therefore, the decisions by actor $i, \mathbf{v}_{\mathbf{i}}$, are stochastic from the point of view of the policy maker, and therefore model output, $f_{N}(u, \overline{\mathbf{u}})$, is a stochastic variable itself.

Again (for one actor) Manne and Richels (1992, ch.4), and (for multiple actors) Manne and Olsen (1996), provide examples of a model of this form in the field of greenhouse gas

reduces stochastic variable to non-stochastic scalars. The inequality $\left.h_{i}\left(z_{i}, x, u, \overline{\mathbf{u}}\right) \leq 0\right)$ represents a set of economic or physical constraints with one or more stochastic components.

${ }^{12}$ The domain and range of the function $f_{M}$ stated explicitly: $f_{M}: D_{Q} \times \bar{D}_{Q} \rightarrow Z \times X$ with $Z=$ $Z_{1} \times \cdots \times Z_{n}$ and $z_{i} \in Z_{i}$. 
emission scenarios. They classify it under the name 'learn then act'. In both models, damage potential associated with a given level of radiative forcing is stochastic.

In long-term models, the situation where actors are aware of the true value of some parameters is not uncommon: in Manne and Richels (1992, ch.4) and Manne and Olsen (1996), for example, it is assumed that the true value of the climate sensitivity parameter will be 'revealed' after some time. This 'revelation' can be interpreted as an abstraction of the better understanding of the climate system and a longer time series of observations after the passage of some time. So the actors, world regions in their models, will observe the true value in the future. But at the base year, only a probability distribution of this variable is known. The ignorance in the base year with respect to the true value of this parameter can be interpreted as the ignorance of a policy maker who uses the model as a basis for decision-making in the base year.

Because $f_{N}(u, \overline{\mathbf{u}})$ is a stochastic variable itself policy maker $Q$ will in theory compare distributions $f_{N}(u, \overline{\mathbf{u}})$ and pick the most preferable one, for example, by choosing $u_{N}^{*} \in D_{Q}$, the one with the highest expected value of his preference function

$$
E_{\overline{\mathbf{u}}}\left[g_{Q}\left(u_{N}^{*}, \overline{\mathbf{u}}, f_{N}\left(u_{N}^{*}, \overline{\mathbf{u}}\right)\right)\right] \geq E_{\overline{\mathbf{u}}}\left[g_{Q}\left(u, \overline{\mathbf{u}}, f_{N}(u, \overline{\mathbf{u}})\right)\right] \quad \text { for all } u \in D_{Q}
$$

Comparison of decisions resulting from application of models $\mathbf{M}$ and $\mathbf{N}$. In general $u_{N}^{*}$ and $u_{M}^{*}$ will not be equal. Examples can be found in several studies in the field of long term greenhouse gas emissions models (Manne and Richels, 1992; Peck and Teisberg, 1993; Manne and Olsen, 1996; Nordhaus and Popp, 1997).

From this analysis we see that model results depend on the assumptions made with respect to the attribution of uncertainty. In sum, the explicit statement about whether actors and/or the policy maker experience uncertainty prevents potential misinterpretation of model results.

\section{Conclusion}

Results from quantitative models in fields where human behavior plays a role are relative, in other words, their correct interpretation depends on (1a) the policy maker for whom the model is developed - in particular, his preferences and decision-making power, and (1b) whether it is meant as a descriptive or prescriptive model.

Corollary 1: in at least some cases potentially significant misinterpretations of model results can be prevented if model developers explicitly state for which policy maker - in particular by describing his preferences and decision-making power - the model is developed for. And whether the results should be interpreted as either prescriptive or descriptive.

Results from quantitative models in fields where human behavior plays a role and the policy maker experiences uncertainty with regard to exogenous variables not under his influence 
are relative with respect to whether or not actors - the model components that represent behavior - experience the uncertainty as well.

Corollary 2: Potentially significant misinterpretation of model results would be prevented when model developers explicitly state which actor experiences what uncertainty in the model with respect to exogenous parameters.

These conclusions are particulary relevant for the use of model results for policy purposes in the field of global, long term greenhouse gas emission reduction strategies. The first corollary is relevant because the global warming issue involves many stakeholders (individuals, regions, sectors and generations) that potentially have fundamentally different preferences and therefore having different opinions about the results from prescriptive modeling efforts (Tol, 1996), and have fundamentally different forms and levels of decision-making power. The second corollary is relevant because decision making is hampered by the uncertainty of scientists and decision-makers with regard to the necessity and consequences of decisions with respects to greenhouse gas emission reductions, partly originating from the uncertainty involved the long time scale of the issue and the chaotic nature of the atmospheric system. 


\section{References}

Alcamo, J., Shaw, R. and Hordijk, L. (1990), The RAINS Model of Acidification in Europe, Kluwer, Dordrecht, the Netherlands.

Arrow, K. J., Cline, W. R., Maler, K.-G., Munasinghe, M., Squitieri, R. and Stiglitz, J. E. (1996), Intertemporal equity, discounting, and economic efficiency, in J. P. Bruce, H. Lee and E. F. Haites, eds, 'Climate Change 1995. Economic and Social Dimensions of Climate Change', Cambridge University Press, chapter 4, pp. 125-144.

Azar, C. (1998), 'Are optimal $\mathrm{CO}_{2}$ emission really optimal?', Environmental and Resource Economics 11(3-4), 301-315. Special issue: 'Frontiers of Environmental \& Resource Economics: Testing the Theories', T. Sterner and J.C.J.M. van den Bergh (eds.).

Bader, P. (1998), Targets and strategies: The role of economic assessments in European climate policy, Technical Report E-98-14, John F. Kennedy School of Government, Cambridge, Ma. Internet: http://environment.harvard.edu/gea/pubs/e\%2D98\%2D14.pdf.

Clark, W. C. and Majone, G. (1985), 'The critical appraisal of scientific enquiries with policy implications', Science, Technology and Human Values 10(3), 6-19.

Fankhauser, S., Tol, R. S. J. and Pearce, D. W. (1997), 'The aggregation of climate change damages: A welfare theoretic approach', Environmental and Resource Economics 10, 249-266.

Gerlagh, R. (1998), The Efficient and Sustainable Use of Environmental Resource Systems, Aula Thesis, Amsterdam, the Netherlands.

Hordijk, L. (1997). Personal communication.

IPCC (1996), Climate Change 1995. Economic and Social Dimensions of Climate Change, Cambridge University Press, Cambridge, UK.

Jaeger, C. C., Renn, O., Rosa, E. A. and Webler, T. (1998), Decision analysis and rational action, in S. Rayner and E. L. Malone, eds, 'Tools for Policy Analysis', Vol. 3 of Human Choice and Climate Change, Batelle Press, Columbus, Ohio, chapter 3, pp. 141-215.

Manne, A. S. (1995), 'The rate of time preference. Implications for the greenhouse debate', Energy Policy 23(4/5), 391-394.

Manne, A. S. and Olsen, T. R. (1996), 'Greenhouse gas abatement - toward Pareto-optimal decisions under uncertainty', Annals of Operations Research 68, 267-279.

Manne, A. S. and Richels, R. G. (1992), Buying Greenhouse Insurance. The Economic Costs of $\mathrm{CO}_{2}$ Emission Limits, The MIT Press, Cambridge, Massachusetts. 
McConnell, K. E. (1997), 'Income and the demand for environmental quality', Environment and Development Economics 2, 383-399.

Neij, L. (1997), 'Use of experience curves to analyse the prospects for diffusion and adoption of renewable energy technology', Energy Policy 23(13), 1099-1107.

Nordhaus, W. D. and Popp, D. (1997), 'What is the value of scientific knowledge? An application to global warming using the PRICE model.', The Energy Journal 18(1), 145.

O'Riordan, T. (1997), 'Review of "Climate change 1995. Economic and social dimensions" (IPCC, 1996)', Environment 39(9), 34-39.

Parson, E. A. (1995), 'Integrated assessment and environmental policy making. In pursuit of usefulness', Energy Policy 23(4/5), 463-475.

Parson, E. A. (1996), 'Three dilemmas in the integrated assessment of climate change', Climatic Change 34, 315-326. Editorial comment.

Pearce, D. W. and Turner, R. K. (1990), Economics of Natural Resources and the Environment, Harvester Wheatsheaf, New York.

Peck, S. C. and Teisberg, T. J. (1993), 'Global warming uncertainties and the value of information: An analysis using CETA', Resource and Energy Economics 15, 71-79.

Perman, R., Ma, Y. and McGilvray, J. (1996), Natural Resource and Environmental Economics, Addison Wesley Longman, New York.

Robinson, J. B. (1992), 'Of maps and territories. The use and abuse of socioeconomic modeling in support of decision making.', Technological Forecasting and Social Change 42, 147-164.

Stewart, T. R. (1997), Forecast value: Descriptive decision studies, in R. W. Katz and A. H. Murphy, eds, 'Economic Value of Wheather and Climate Forecasts', Cambridge University Press, Cambridge, UK, chapter 5, pp. 147-181.

Tol, R. S. J. (1996), The social cost controversy: A personal appraisal, in A. Sors, A. Liberatore, S. Funtowicz, J. C. Hourcade and J. L. Fellous, eds, 'Proceedings of the International Symposium "Prospects for Integrated Environmental Assessment: Lessons Learnt From the Case of Climate Change"', European Commision DG XII, Brussels, Belgium, pp. 35-40. Toulouse, France, October 24-26, 1996. 


\section{A Description of variables}

Table 1: Description of variables (in order of appearance).

\begin{tabular}{l|l|l}
\hline$r$ & Model output & $r \in O$ \\
$u$ & Decision by policymaker $P$ & $u \in D_{P}$ \\
$\bar{u}$ & Exogenous variable not controllable by policymaker $P$ & $\bar{u} \in \bar{D}_{P}$ \\
$\overline{\mathbf{u}}$ & Stochastic exogenous variable not controllable by policymaker $P$ & $\overline{\mathbf{u}} \in \bar{D}_{P}$ \\
$x$ & Vector of endogenous variables & $x \in X$ \\
$z_{i}$ & Potential decision by actor $i$ & $z_{i} \in Z_{i}$ \\
$v_{i}$ & Optimal decision by actor $i$ & $v_{i} \in Z_{i}$ \\
$\alpha_{i}$ & Chance level & $\alpha_{i} \in[0, \ldots, 1]$ \\
$x_{k}$ & Vector of endogenous variables when value of $\overline{\mathbf{u}}$ is $\bar{u}_{k}$ & $x_{k} \in X$ \\
$z_{i k}$ & Potential decision by actor $i$ when value of $\overline{\mathbf{u}}$ is $\bar{u}_{k}$ & $z_{i k} \in Z_{i}$ \\
$v_{i k}$ & Optimal decision by actor $i$ when value of $\overline{\mathbf{u}}$ is $\bar{u}_{k}$ & $v_{i k} \in Z_{i}$ \\
\hline
\end{tabular}

